\title{
The association of serum total bile acid with non-alcoholic fatty liver disease in Chinese adults: a cross sectional study
}

\author{
Ziyu Zhang ${ }^{1 \dagger}$, Wen Dai ${ }^{1 \dagger}$, Shuwei Weng ${ }^{1}$, Mengdie Luo ${ }^{1}$, Jiahao Fu², John A. Zadroga ${ }^{3}$, Stefano Spolitu ${ }^{4}$ and \\ Daoquan Peng ${ }^{1 *}$
}

\begin{abstract}
Background: Non-alcoholic fatty liver disease (NAFLD) is currently the major cause of chronic liver disease globally. Bile acids (BAs) have emerged as relevant signaling molecules that are associated with NAFLD development. This study was aimed to examine the association of serum total bile acids (TBAs) with NAFLD in a large population of Chinese subjects.
\end{abstract}

Methods: This cross sectional study recruited 152,336 participants from the Second Xiangya Hospital, China. NAFLD was diagnosed based on the presence of hepatic steatosis on ultrasonography, without significant alcohol consumption and other known causes for chronic liver disease. A multivariate logistic regression model was used to test for the association of serum TBAs with NAFLD, adjusting for conventional risk factors of NAFLD.

Results: A total of $27.4 \%$ of the participants had NAFLD. Patients with NAFLD had slightly higher TBA levels than those without, 3.4 vs. $3.0 \mu \mathrm{mol} / \mathrm{L}(p<0.001)$. However, TBA levels were not associated with NAFLD in the multivariate logistic regression model, which adjusted for age, gender and other acknowledged risk factors for NAFLD (OR $=1.00 .95 \% \mathrm{Cl}: 1.00-1.00, p=0.797)$.

Conclusions: We found that the serum TBA levels were not associated with NAFLD. Future studies in a large population, focusing on serum BA composition may improve the understating of the role of BAs in NAFLD.

Keywords: Non-alcoholic fatty liver disease, Bile acid, Farnesoid X receptor, Takeda G-protein-coupled receptor 5, Type 2 diabetes, Ziyu Zhang and Wen Dai contributed equally to this work and were listed co-first authors.

\section{Background}

Non-alcoholic fatty liver disease (NAFLD) is the hepatic manifestation of metabolic syndrome and is currently the major cause of chronic liver disease globally [1-3]. The estimated prevalence of NAFLD worldwide is approximately $25 \%$ in the general population [4]. NAFLD includes a broad clinical and histological spectrum ranging from simple hepatic steatosis (HS) to non-alcoholic steatohepatitis (NASH), with varying degrees of inflammation and fibrosis, which can progress to cirrhosis. It has been reported that approximately $20-30 \%$ of

\footnotetext{
* Correspondence: daoquanpeng2019@sina.com

†Ziyu Zhang and Wen Dai contributed equally to this work.

'Department of Cardiology, The Second Xiangya Hospital, Central South

University, 139 Middle Renmin Road, Changsha 410011, China

Full list of author information is available at the end of the article
}

subjects with HS would develop NASH [5]. Moreover, NASH has become the leading cause of liver failure and the major indication for liver transplantation [6-9].

Bile acids (BAs) are amphipathic steroid acids that are derived from hepatic cholesterol catabolism in a series of enzyme-catalyzed reactions [10]. Upon synthesis, BAs are subsequently secreted and drained into the gallbladder for temporary storage via the biliary ducts. Postprandial contraction of the gallbladder releases BAs to the duodenum. In the intestine, BAs facilitate the emulsification and absorption of dietary lipids, as well as lipophilic vitamins [10]. After reaching the terminal ileum, BAs are almost completely $(95 \%)$ reabsorbed and recirculated to the liver via the portal vein. The reabsorbed BAs are secreted back into the bile ducts together with newly synthesized BAs. This process is known as bile acid enterohepatic

(c) The Author(s). 2020 Open Access This article is distributed under the terms of the Creative Commons Attribution 4.0 International License (http://creativecommons.org/licenses/by/4.0/), which permits unrestricted use, distribution, and 
circulation, which allows for efficient recovery and reuse of the vast majority of BAs [10]. However, a small fraction of reabsorbed BAs bypass enterohepatic circulation and reach the systemic circulation [10].

Interestingly, circulating BAs have emerged as relevant signaling molecules, which act on hepatic and extrahepatic tissues to regulate lipid and carbohydrate metabolic pathways, as well as energy homeostasis, via activation or modulation of bile acid receptors, such as the farnesoid $\mathrm{X}$ receptor (FXR) and Takeda G-protein-coupled receptor 5 (TGR5) [11-14]. It has been reported that the BA-FXR/ TGR5 pathways regulate hepatic lipid and glucose metabolism, as well as inflammatory activity [15-19].

Moreover, recent studies have shown that blood BAs are closely associated with NAFLD in humans. Serum total bile acid (TBA) levels in NAFLD patients $(n=16)$ were approximately 3 times higher than those of healthy controls $(n=11)$ [20]. Similarly, Rebecca et al. found that patients with type 2 diabetes (T2D), a major risk factor for NAFLD, had a nearly two-fold elevation in their plasma TBA [21].

A major limitation of these studies was their small sample sizes and to our knowledge, the association between serum TBAs and NAFLD has not been examined in a large-scale epidemiologic study before. Thus, this study was aimed to evaluate whether serum TBA levels were independently associated with NAFLD in a large population of Chinese subjects.

\section{Methods}

\section{Study design and population}

In this cross-sectional study, participants were recruited from the Health Management Center, The Second Xiangya Hospital, Changsha, China, from 2010 to 2017. We included adult participants who were scheduled to receive systemic medical evaluation, including subjective medical history, physical exam, blood chemistries, electrocardiogram and abdominal ultrasound. Subjects were excluded if they 1) were less than 18 years old; 2) reported excess alcohol consumption or a history of viral hepatitis, autoimmune hepatitis, or other known causes of chronic liver disease. 152,336 subjects were ultimately included in this study. This study was approved by the ethics committee institutional review board of The Second Xiangya Hospital of Central South University. The study was carried out in accordance with the 1975 Declaration of Helsinki and pertinent regulations. Informed written consent was obtained from all participants.

\section{Data collection}

Demographic characteristics, medical histories, blood chemistries and abdominal ultrasound data were gathered for all participants at the time of enrollment. Specific blood chemistries included fasting serum BAs, glucose and lipids, as well as liver and renal function tests. As previously described [22, 23], blood samples were drawn by venipuncture after overnight fasting. The blood specimens were processed and measured at the central laboratory in The Second Xiangya hospital, which is standardized and certified. An automatic biochemistry analyzer (Hitachi 7360; Hitachi Ltd., Tokyo, Japan) and commercially available kits were used to measure serum biochemistries according to accompanying manuals. Specifically, serum BAs were measured using enzymatic assay [24].

NAFLD was diagnosed based on the evidence of fatty liver upon abdominal ultrasonography and the exclusion of other known etiology of chronic liver diseases [25]. Hypertension was referred to as blood pressure $\geq 140 / 90$ $\mathrm{mmHg}$ in more than two measurements and/or the requirement of or treatment with anti-hypertension drugs [26]. Diabetes was diagnosed as the existence of fasting serum glucose levels $\geq 7.0 \mathrm{mmol} / \mathrm{L}$, and/or random serum glucose $\geq 11.1 \mathrm{mmol} / \mathrm{L}$, and/or 2 -h post-prandial serum glucose $\geq 11.1 \mathrm{mmol} / \mathrm{L}$ on oral glucose tolerance test (OGTT) in multiple measurements and/or the requirement of treatment with hypoglycemic agents [27]. Patients were diagnosed with coronary artery disease (CAD) based on the clinical manifestation, such as chest pain, and evidences indicating myocardial ischemia $[28,29]$.

\section{Statistical analysis}

Numerical variables were expressed as the mean (standard deviation) or as medians (Q1-Q3 quartiles), depending on the pattern of data distribution. Categorical variables were expressed as percentages (numbers). Differences in numerical variables between groups were analyzed by the independent $t$ test, analysis of the variance (ANOVA), Mann-Whitney $U$ test or Kruskal-Wallis $H$ test, as appropriate. Differences in categorical variables were analyzed by the chi-square test. Logistic regression analyses were performed to examine the association of serum TBA with NAFLD and T2D. SPSS software (version 20.0; SPSS Inc., Cary, Chicago, USA) was used to perform the statistical analyses. For all analyses, two-tailed $p$ values $<0.05$ were considered statistically significant.

\section{Results \\ Clinical characteristics}

The demographic and clinical characteristics of the study population were shown in Table 1 . The mean age and body mass index (BMI) of participants was 45 years old and $24.0 \mathrm{~kg} / \mathrm{m}^{2}$, respectively, and $54.2 \%(82,533 / 152,336)$ were males. $27.4 \%(41,771 / 152,336)$ of individuals were diagnosed with NAFLD. We also found 19.5\% (29,767/152, $336), 3.7 \%(5623 / 152,336)$ and $1.3 \%(1920 / 152,336)$ of participants had hypertension, T2D and CAD, respectively. Individuals with NAFLD were noted to be older, have a higher BMI and increased prevalence of hypertension, T2D and CAD than those without (all $p<0.001$ ). 
Table 1 Demographic and clinic characteristics of the study population

\begin{tabular}{|c|c|c|c|c|}
\hline & Total $(n=152,336)$ & With NAFLD $(n=41,771)$ & Without NAFLD $(n=110,565)$ & $p$ \\
\hline \multicolumn{5}{|l|}{ Clinical characteristics } \\
\hline Age, years & $45(13)$ & $48(12)$ & $44(14)$ & $<.001$ \\
\hline Male percentage, \% (n) & $54.2 \%(82,533)$ & $75.4 \%(31,510)$ & $46.1 \%(51,023)$ & $<.001$ \\
\hline $\mathrm{BMI}, \mathrm{kg} / \mathrm{m}^{2}$ & $24.0(3.3)$ & $27.1(2.8)$ & $22.9(2.8)$ & $<.001$ \\
\hline Systolic pressure, mm Hg & $120(17)$ & $128(16)$ & $117(17)$ & $<.001$ \\
\hline Diastolic pressure, $\mathrm{mm} \mathrm{Hg}$ & $76(11)$ & $81(11)$ & $74(10)$ & $<.001$ \\
\hline Hypertension, \% (n) & $19.5 \%(29,767)$ & $33.8 \%(14,129)$ & $14.1 \%(15,638)$ & $<.001$ \\
\hline T2D, \% (n) & $3.7 \%(5623)$ & $6.9 \%(2870)$ & $2.5 \%(2753)$ & $<.001$ \\
\hline$C A D, \%(n)$ & $1.3 \%(1920)$ & $1.8 \%(733)$ & $1.1 \%(1187)$ & $<.001$ \\
\hline \multicolumn{5}{|l|}{ Biochemical parameters } \\
\hline TBA, $\mu \mathrm{mol} / \mathrm{L}$ & $2.0(3.1-5.0)$ & $3.4(2.3-5.4)$ & $3.0(1.9-4.8)$ & $<.001$ \\
\hline AST, U/L & $23(17)$ & $26(14)$ & $21(17)$ & $<.001$ \\
\hline$A L T, U / L$ & $26(26)$ & $37(28)$ & $22(24)$ & $<.001$ \\
\hline BUN, mmol/L & $5.0(1.4)$ & $5.2(1.4)$ & $5.0(1.5)$ & $<.001$ \\
\hline $\mathrm{Cr}, \mu \mathrm{mol} / \mathrm{L}$ & $67.3(22.6)$ & $71.9(19.0)$ & $65.6(23.6)$ & $<.001$ \\
\hline$U \mathrm{~A}, \mu \mathrm{mol} / \mathrm{L}$ & $312.3(83.1)$ & $359.3(81.2)$ & $294.6(75.8)$ & $<.001$ \\
\hline $\mathrm{FBG}, \mathrm{mmol} / \mathrm{L}$ & $5.04(1.29)$ & $5.48(1.76)$ & $4.90(1.01)$ & $<.001$ \\
\hline OGTT-2 h BG †, mmol/L & $7.10(3.07)$ & $7.74(3.45)$ & $6.74(2.77)$ & $<.001$ \\
\hline $\mathrm{TC}, \mathrm{mmol} / \mathrm{L}$ & $4.66(0.93)$ & $4.92(1.00)$ & $4.56(0.88)$ & $<.001$ \\
\hline $\mathrm{HDLC}, \mathrm{mmol} / \mathrm{L}$ & $1.31(0.30)$ & $1.16(0.24)$ & $1.37(0.30)$ & $<.001$ \\
\hline $\mathrm{LDLC}, \mathrm{mmol} / \mathrm{L}$ & $2.67(0.77)$ & $2.81(0.83)$ & $2.62(0.74)$ & $<.001$ \\
\hline $\mathrm{RLP}-\mathrm{C}, \mathrm{mmol} / \mathrm{L}$ & $0.68(0.61)$ & $0.95(0.88)$ & $0.57(0.43)$ & $<.001$ \\
\hline $\mathrm{TG}, \mathrm{mmol} / \mathrm{L}$ & $1.24(0.86-1.86)$ & $1.87(1.33-2.74)$ & $1.07(0.78-1.53)$ & $<.001$ \\
\hline
\end{tabular}

Data are shown as mean (standard deviation), median (Q1-Q3 quartiles), or percentages (n). $P$ values from analysis of the independent $\mathrm{t}$ test, Mann-Whitney $U$ test, or chi-square test. Two-tailed $p<0.05$ was considered statistically significant. BMI body mass index, NALFD non-alcoholic fatty liver disease, T2D type 2 diabetes, $C A D$ coronary artery disease, TBA total bile acid, FBG fasting blood glucose, $A S T$ aspartate aminotransferase, $A L T$ alanine aminotransferase, BUN blood urea nitrogen, $C r$ creatinine, UA uric acid, TC total cholesterol, LDL-C LDL cholesterol, HDL-C HDL cholesterol, RLP-C remnant lipoprotein cholesterol, TG triglyceride. OGTT-2 $\mathrm{h} B G$ oral glucose tolerance test $2 \mathrm{~h}$-post blood glucose. +7870 individuals had the OGTT- $2 \mathrm{~h}$ data

\section{Serum TBA levels}

Consistent with previous findings [30], the distribution of serum TBA levels was positively skewed in both male and female populations in our study (Figure 1). The median level of TBA was $3.4 \mu \mathrm{mol} / \mathrm{L}$ in males, which was significantly higher than the level of $2.8 \mu \mathrm{mol} / \mathrm{L}$ in females $(p<0.001)$. Moreover, this was applied to all age strata in the study population (Table 2). Currently, the reference range of TBA is less than $10 \mu \mathrm{mol} / \mathrm{L}$ for both males and females in the clinical setting. According to this criterion, $6.2 \%$ of males and $3.5 \%$ of females had elevated TBA in this study.

\section{Serum TBA and NAFLD}

Patients with NAFLD were noted to have slightly higher TBA levels than those without ( 3.4 vs. $3.0 \mu \mathrm{mol} / \mathrm{L}, p<0.001$ ) (Table 1). We then calculated the prevalence of NAFLD categorized by TBA quintiles and we found that the prevalence of NAFLD increased with the elevation in TBA levels. The 5 th quintile (Q5) group had the highest (31.3\%) while the 1st quintile (Q1) group had the lowest prevalence (20.8\%) of NAFLD (Table 3). Furthermore, we used a multivariate logistic regression model to test whether serum TBA was associated with NAFLD after adjusting for age, gender, BMI and other acknowledged risk factors for NAFLD. After this adjustment, we found that the association between serum TBA level and NAFLD was lost (Table 4).

\section{Serum TBA and T2D}

Since NAFLD is closely associated with T2D and insulin resistance is considered one of the major cause for NAFLD development, we also checked the association of serum TBA with T2D. Similarly, we found that the prevalence of T2D rose with increasing TBA levels. Notably, the Q5 group had the highest and Q1 group had the lowest prevalence of T2D, $5.1 \%$ vs. $2.0 \%$, respectively (Table 3). However, in our multivariate logistic regression analysis, serum TBA was not associated with T2D in the model controlling for age, gender, BMI and known T2D risk factors (Table 5). 


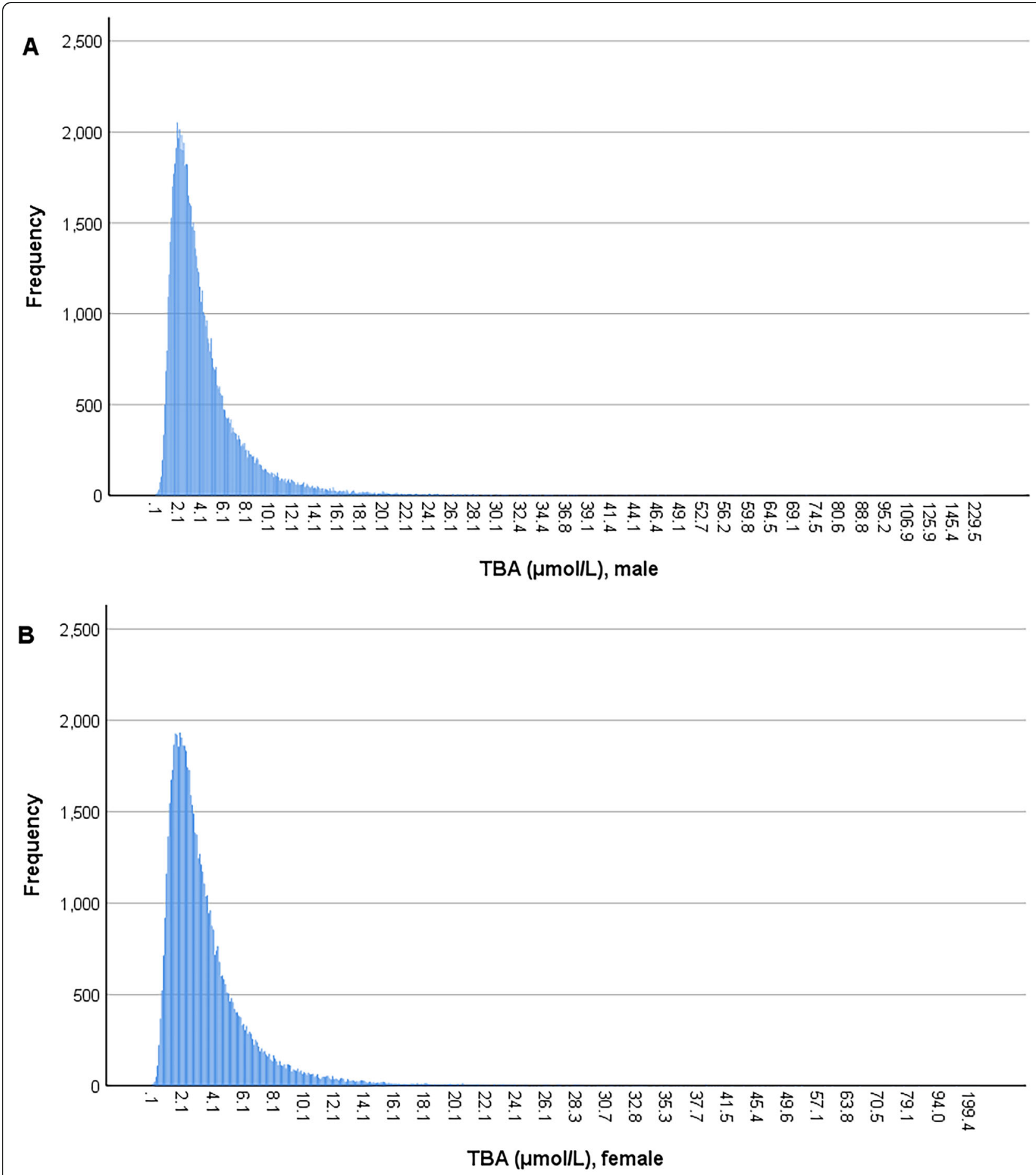

Fig. 1 Distribution of serum total bile acid levels in males and females. a. distribution of serum total bile acid levels in males, $\mathbf{b}$. distribution of serum total bile acid levels in females

\section{Discussion}

The understandings of the function and effect of BA modulated receptors in the liver, such as FXR, suggest the potential role of BAs in NAFLD development. However, in this study, we found that serum TBA levels were not independently associated with NAFLD or T2D when controlling for age, gender, BMI and additional risk factors.

BAs are a group of diverse amphipathic steroids, rather than a single type of molecule, derived from the 
Table 2 Gender and age stratum specific median levels of serum TBA

\begin{tabular}{|c|c|c|c|c|c|}
\hline \multirow[t]{2}{*}{ Age } & \multicolumn{2}{|l|}{ Male } & \multicolumn{2}{|l|}{ Female } & \multirow[t]{2}{*}{$p$} \\
\hline & $n$ & $\mathrm{TBA}, \mu \mathrm{mol} / \mathrm{L}$ & $n$ & $\mathrm{TBA}, \mu \mathrm{mol} / \mathrm{L}$ & \\
\hline$<20$ & 189 & $2.9(1.9-4.6)$ & 179 & $3.0(1.8-5.1)$ & $<0.01$ \\
\hline $20 \sim 29$ & 9099 & $3.4(2.2-5.4)$ & 10,283 & $2.9(1.9-4.5)$ & $<0.01$ \\
\hline $30 \sim 39$ & 18,094 & $3.2(2.1-5.1)$ & 16,062 & $2.6(1.7-4.1)$ & $<0.01$ \\
\hline $40 \sim 49$ & 22,738 & $3.3(2.1-5.2)$ & 18,851 & $2.7(1.7-4.2)$ & $<0.01$ \\
\hline $50 \sim 59$ & 18,852 & $3.4(2.2-5.4)$ & 15,243 & $2.9(1.9-4.7)$ & $<0.01$ \\
\hline $60 \sim 69$ & 8377 & $3.6(2.4-5.8)$ & 6445 & $3.2(2.1-5.2)$ & $<0.01$ \\
\hline$\geq 70$ & 5184 & $4.2(2.8-6.9)$ & 2740 & $3.9(2.5-6.2)$ & $<0.01$ \\
\hline Total & 82,533 & $3.4(2.2-5.4)$ & 69,803 & $2.8(1.8-4.5)$ & $<0.01$ \\
\hline
\end{tabular}

Data are shown as median (Q1-Q3 quartiles). $P$ values from analysis of the Kruskal-Wallis $\mathrm{H}$ test. Two-tailed $p<0.05$ was considered statistically significant. TBA total bile acid catabolism of cholesterol in hepatocytes in a process that involves two principal pathways [10]. The classical pathway, which generates the majority of BAs, is regulated by the rate-limiting enzyme, cholesterol $7 \alpha$-hydroxylase (CYP7A1). The alternative pathway is initially catalyzed by sterol-27-hydroxlase (CYP27A1), followed by BA hydroxylation through oxysterol $7 \alpha$-hydroxylase (CYP7B1). These two pathways account for the synthesis of the primary BAs, namely chenodeoxycholic acid (CDCA) and cholic acid (CA). They, in turn, are modified by gut microbiota to yield lithocholic acid (LCA) and deoxycholic acid (DCA) respectively, which represent two major type of secondary BAs.

Different types of BAs differ in in their physicochemical properties. For example, whereas CDCA is a potent agonist of FXR, DCA and LCA have been suggested as partial antagonists of FXR [10]. This suggests they may have a distinct regulatory effect on metabolism. Recent findings indicated that alterations in circulating BA composition were closely associated with NAFLD and T2D. $\mathrm{Na}$ et al. measured the serum BA composition of NAFLD patients and healthy controls and found that the

Table 3 Basic characteristics of the study population categorized by TBA quintiles

\begin{tabular}{|c|c|c|c|c|c|c|}
\hline & \multicolumn{5}{|c|}{ Serum TBA quintiles } & \multirow[t]{2}{*}{$P$} \\
\hline & $\mathrm{Q} 1(n=31,267)$ & Q2 $(n=30,136)$ & Q3 $(n=31,298)$ & Q4 $(n=29,245)$ & Q5 $(n=30,390)$ & \\
\hline \multicolumn{7}{|l|}{ Clinical characteristics } \\
\hline Age, years & $44(12)$ & $45(13)$ & $45(13)$ & $46(14)$ & $48(15)$ & $<.001$ \\
\hline Male percentage, \% (n) & $42.5 \%(13,284)$ & $52.0 \%(15,679)$ & $55.7 \%(17,428)$ & $58.6 \%(17,129)$ & $62.6 \%(19,013)$ & $<.001$ \\
\hline $\mathrm{BMI}, \mathrm{kg} / \mathrm{m} 2$ & $23.7(3.3)$ & $24.0(3.3)$ & $24.1(3.4)$ & $24.2(3.4)$ & $24.3(3.4)$ & $<.001$ \\
\hline Systolic pressure, mm Hg & $117(17)$ & $119(17)$ & $120(17)$ & $121(17)$ & $123(18)$ & $<.001$ \\
\hline Diastolic pressure, mm Hg & $74(11)$ & $75(11)$ & $76(11)$ & $76(11)$ & $77(11)$ & $<.001$ \\
\hline NALFD, \% (n) & $20.8 \%(6512)$ & $26.0 \%(7840)$ & $28.8 \%(9012)$ & $30.4 \%(8895)$ & $31.3 \%(9512)$ & $<.001$ \\
\hline Hypertension, \% (n) & $14.7 \%(4581)$ & $17.6 \%(5309)$ & $19.3 \%(6028)$ & $21.9 \%(6403)$ & $24.5 \%(7446)$ & $<.001$ \\
\hline T2D, \% (n) & $2.0 \%(630)$ & $3.1 \%(931)$ & $3.7 \%(1161)$ & $4.6 \%(1358)$ & $5.1 \%(1543)$ & $<.001$ \\
\hline$C A D, \%(n)$ & $0.9 \%(269)$ & $0.9 \%(282)$ & $1.2 \%(387)$ & $1.4 \%(407)$ & $1.9 \%(575)$ & $<.001$ \\
\hline Biochemical parameters & & & & & & $<.001$ \\
\hline AST, U/L & $20(7)$ & $21(9)$ & $22(9)$ & $23(10)$ & $26(32)$ & $<.001$ \\
\hline $\mathrm{ALT}, \mathrm{U} / \mathrm{L}$ & $22(14)$ & $25(18)$ & $26(20)$ & $27(22)$ & $32(44)$ & $<.001$ \\
\hline $\mathrm{BUN}, \mathrm{mmol} / \mathrm{L}$ & $4.8(1.4)$ & $4.9(1.4)$ & $5.1(1.5)$ & $5.1(1.5)$ & $5.1(1.5)$ & $<.001$ \\
\hline $\mathrm{Cr}, \mu \mathrm{mol} / \mathrm{L}$ & $64.6(21.7)$ & $66.7(21.5)$ & 67.9 (26.6) & $68.3(21.7)$ & $69.2(20.5)$ & $<.001$ \\
\hline UA, $\mu \mathrm{mol} / \mathrm{L}$ & $299.1(80.3)$ & $310.4(82.6)$ & $314.7(83.2)$ & $317.3(83.8)$ & $320.6(84.2)$ & $<.001$ \\
\hline $\mathrm{FBG}, \mathrm{mmol} / \mathrm{L}$ & $4.90(1.01)$ & $4.98(1.18)$ & $5.04(1.31)$ & $5.11(1.41)$ & $5.16(1.49)$ & $<.001$ \\
\hline $\mathrm{TC}, \mathrm{mmol} / \mathrm{L}$ & $4.65(0.91)$ & $4.66(0.92)$ & $4.66(0.92)$ & 4.67 (0.95) & $4.66(0.97)$ & 0.103 \\
\hline $\mathrm{HDLC}, \mathrm{mmol} / \mathrm{L}$ & $1.35(0.29)$ & $1.32(0.29)$ & $1.30(0.29)$ & $1.29(0.30)$ & $1.28(0.31)$ & $<.001$ \\
\hline $\mathrm{LDLC}, \mathrm{mmol} / \mathrm{L}$ & $2.70(0.76)$ & $2.69(0.77)$ & $2.68(0.77)$ & 2.67 (0.78) & $2.62(0.78)$ & $<.001$ \\
\hline $\mathrm{RLP}-\mathrm{C}, \mathrm{mmol} / \mathrm{L}$ & $0.60(0.47)$ & $0.65(0.56)$ & $0.68(0.60)$ & $0.71(0.66)$ & $0.76(0.74)$ & $<.001$ \\
\hline $\mathrm{TG}, \mathrm{mmol} / \mathrm{L}$ & $1.11(0.80-1.61)$ & $1.21(0.84-1.81)$ & $1.25(0.87-1.90)$ & $1.30(0.89-1.97)$ & $1.34(0.92-2.06)$ & $<.001$ \\
\hline
\end{tabular}

Data are shown as mean (standard deviation), median (Q1-Q3 quartiles), or percentages (n). $P$ values from analysis of the ANOVA, Kruskal-Wallis $H$ test, or chisquare test. Two-tailed $p<0.05$ was considered statistically significant. $B M I$ body mass index, NALFD non-alcoholic fatty liver disease, $T 2 D$ type 2 diabetes, CAD coronary artery disease, TBA total bile acid, FBG fasting blood glucose, $A S T$ aspartate aminotransferase, $A L T$ alanine aminotransferase, $B U N$ blood urea nitrogen, $C r$ creatinine, UA uric acid, TC total cholesterol, LDL-C LDL cholesterol, HDL-C HDL cholesterol, RLP-C remnant lipoprotein cholesterol, TG triglyceride 
Table 4 The association of serum TBA with NAFLD by multivariate logistic regression analysis

\begin{tabular}{llll}
\hline Variables & OR & $95 \% \mathrm{Cl}$ & $\mathrm{p}$ \\
\hline Age & 1.02 & $1.01-1.02$ & $<0.001$ \\
Gender (male vs. female) & 1.47 & $1.42-1.52$ & $<0.001$ \\
BMI & 1.59 & $1.58-1.60$ & $<0.001$ \\
History of hypertension (with vs. without) & 1.28 & $1.23-1.33$ & $<0.001$ \\
History of T2D (with vs. without) & 1.58 & $1.48-1.70$ & $<0.001$ \\
TC & 0.98 & $0.92-1.04$ & 0.501 \\
LDL-C & 1.39 & $1.31-1.48$ & $<0.001$ \\
HDL-C & 0.38 & $0.34-0.42$ & $<0.001$ \\
TG & 1.35 & $1.31-1.39$ & $<0.001$ \\
TBA & 1.00 & $1.00-1.00$ & 0.797 \\
\hline
\end{tabular}

$P$ values were from multivariate logistic regression. Two-tailed $p<0.05$ was considered statistically significant. TBA total bile acid, NAFLD non-alcoholic fatty liver disease, $B M I$ body mass index, T2D type 2 diabetes, $T C$ total cholesterol, LDL-C LDL cholesterol, HDL-C HDL cholesterol, TG triglyceride

percentage of FXR antagonistic DCA was increased, while the agonistic CDCA was decreased in patients with NAFLD compared to healthy subjects [20]. Rebecca et al. showed that increased plasma levels of 12ahydroxylated BA were associated with insulin resistance in both T2D patients and healthy subjects [21]. These interesting findings suggest that the alterations in $\mathrm{BA}$ composition rather than quantitative changes in TBA levels are more relevant to metabolic homeostasis.

Currently, no convenient/applicable serum BA composition assessment techniques are available in non-academic clinics. So far, penetration of "classical" laboratory methods, including high-performance liquid chromatography (HPLC), liquid chromatography-mass spectrometry (LCMS) and gas chromatography-mass spectrometry (GCMS), into the clinics is minimal due to the need of expensive

Table $\mathbf{5}$ The association of serum TBA with T2D by multivariate logistic regression analysis

\begin{tabular}{llll}
\hline Variables & OR & $95 \% \mathrm{Cl}$ & $\mathrm{p}$ \\
\hline Age & 1.07 & $1.07-1.07$ & $<0.001$ \\
Gender (male vs. female) & 1.33 & $1.25-1.42$ & $<0.001$ \\
BMI & 1.01 & $1.00-1.02$ & 0.136 \\
History of hypertension (with vs. without) & 1.61 & $1.52-1.72$ & $<0.001$ \\
History of NAFLD (with vs. without) & 1.73 & $1.62-1.85$ & $<0.001$ \\
TC & 1.04 & $0.97-1.11$ & 0.253 \\
LDL-C & 0.88 & $0.82-0.95$ & $<0.001$ \\
HDL-C & 0.34 & $0.29-0.39$ & $<0.001$ \\
TG & 1.06 & $1.04-1.09$ & $<0.001$ \\
TBA & 1.00 & $1.00-1.01$ & 0.178
\end{tabular}

$P$ values were from multivariate logistic regression. Two-tailed $p<0.05$ was considered statistically significant. TBA total bile acid, NAFLD non-alcoholic fatty liver disease, $B M I$ body mass index, $T 2 D$ type 2 diabetes, $T C$ total cholesterol, LDL-C LDL cholesterol, HDL-C HDL cholesterol, TG triglyceride equipment and time-consuming protocols [24]. Because of that, we had to resort to the conventional enzymatic assessment of blood TBA in this study, which is widely used in clinical settings

In addition to this, we acknowledge several other limitations to our study. Due to the nature of cross-sectional studies, we were not able to follow participants to determine the predictive values of serum TBA levels in NAFLD development. Besides, we only measured the fasting serum BA levels. The role of postprandial BA levels in metabolism should also be studied in the future.

\section{Conclusion}

Serum TBA was not associated with NAFLD. Future studies in a large population focusing on the serum BA composition may strengthen our understating of the role of BAs in NAFLD development and progression.

\section{Abbreviations}

ANOVA: Analysis of the variance; BAs: Bile acids; BMl: Body mass index; CA: Cholic acid; CAD: Coronary artery disease; CDCA: Chenodeoxycholic acid; CYP27A1: Sterol-27-hydroxlase; CYP7A1: Cholesterol 7a-hydroxylase; CYP7B1: Oxysterol 7a-hydroxylase; DCA: Deoxycholic acid; FXR: Farnesoid X receptor; GC-MS: Gas chromatography-mass spectrometry; HPLC: Highperformance liquid chromatography; HS: Hepatic steatosis; LCA: Lithocholic acid; LC-MS: Liquid chromatography-mass spectrometry; NAFLD: Nonalcoholic fatty liver disease; NASH: Non-alcoholic steatohepatitis; OGTT: Oral glucose tolerance test; T2D: Type 2 diabetes; TBA: Total bile acid;

TGR5: Takeda G-protein-coupled receptor 5

\section{Acknowledgments}

None.

\section{Authors' contributions}

Conceived and designed the study: DP. Performed the study: ZZ, WD, SW and ML. Analyzed the data: ZZ, WD, JF and JAZ. Wrote the paper: ZZ, WD, $J A Z, S S$ and DP. All authors reviewed drafts and approved the final version of the manuscript.

\section{Funding}

This work was supported by the National Natural Science Foundation of China [No. 81870336 and 81670426 to Daoquan Peng]; the Fundamental Research Funds for the Central University of Central South University [No. $2019 z z t s 805$ to Ziyu Zhang].

\section{Availability of data and materials}

The datasets used and/or analyzed during the current study are available from the corresponding author on reasonable request.

\section{Ethics approval and consent to participate}

This study was approved by the ethics committee review board of The Second Xiangya Hospital of Central South University. Informed written consent was obtained from all participants.

Consent for publication

Not applicable.

\section{Competing interests}

The authors declare that they have no competing interests.

\section{Author details}

'Department of Cardiology, The Second Xiangya Hospital, Central South University, 139 Middle Renmin Road, Changsha 410011, China. ${ }^{2}$ National Key Laboratory of Human Factors Engineering, 1 west Yuanmin Yuan Road, Beijing 100094, China. ${ }^{3}$ Rowan University School of Osteopathic Medicine, 1 
Medical Center Drive, Stratford, NJ 08084-1501, USA. ${ }^{\text {Department of }}$ Medicine, Columbia University, 630 west 168th street, New York 10032, USA.

Received: 2 October 2019 Accepted: 24 January 2020

Published online: 04 February 2020

\section{References}

1. Araujo AR, Rosso N, Bedogni G, Tribelli C, Bellentani S. Global epidemiology of non-alcoholic fatty liver disease/non-alcoholic steatohepatitis: what we need in the future. Liver Int. 2018;38(Suppl 1):47-51.

2. Li J, Zou B, Yeo YH, Feng Y, Xie X, Lee DH, et al. Prevalence, incidence, and outcome of non-alcoholic fatty liver disease in Asia, 1999-2019: a systematic review and meta-analysis. Lancet Gastroenterol Hepatol. 2019;4(5):389-98.

3. Fan JG. Epidemiology of alcoholic and nonalcoholic fatty liver disease in China. J Gastroenterol Hepatol. 2013;28(Suppl 1):11-7.

4. Satapathy SK, Sanyal AJ. Epidemiology and natural history of nonalcoholic fatty liver disease. Semin Liver Dis. 2015;35(3):221-35.

5. Rinella ME. Nonalcoholic fatty liver disease: a systematic review. JAMA. 2015; 313(22):2263-73

6. Cholankeril G, Wong RJ, Hu M, Perumpail RB, Yoo ER, Puri P, et al. Liver transplantation for nonalcoholic Steatohepatitis in the US: temporal trends and outcomes. Dig Dis Sci. 2017;62(10):2915-22.

7. Doycheva I, Issa D, Watt KD, Lopez R, Rifai G, Alkhouri N. Nonalcoholic Steatohepatitis is the Most rapidly increasing indication for liver transplantation in young adults in the United States. J Clin Gastroenterol. 2018;52(4):339-46.

8. Doycheva I, Watt KD, Alkhouri N. Nonalcoholic fatty liver disease in adolescents and young adults: the next frontier in the epidemic. Hepatol. 2017:65(6):2100-9.

9. Chedid MF. Nonalcoholic Steatohepatitis: the second leading indication for liver transplantation in the USA. Dig Dis Sci. 2017;62(10):2621-2.

10. Shapiro H, Kolodziejczyk AA, Halstuch D, Elinav E. Bile acids in glucose metabolism in health and disease. J Exp Med. 2018;215(2):383-96.

11. Schaap FG, Trauner M, Jansen PL. Bile acid receptors as targets for drug development. Nat Rev Gastroenterol Hepatol. 2014;1 1(1):55-67.

12. Arab JP, Karpen SJ, Dawson PA, Arrese M, Trauner M. Bile acids and nonalcoholic fatty liver disease: molecular insights and therapeutic perspectives. Hepatol. 2017;65(1):350-62

13. Copple BL, Li T. Pharmacology of bile acid receptors: evolution of bile acids from simple detergents to complex signaling molecules. Pharmacol Res. 2016;104:9-21

14. Li T, Chiang JY. Bile acid signaling in metabolic disease and drug therapy. Pharmacol Rev. 2014;66(4):948-83.

15. Wang YD, Chen WD, Yu D, Forman BM, Huang W. The G-protein-coupled bile acid receptor, Gpbar1 (TGR5), negatively regulates hepatic inflammatory response through antagonizing nuclear factor kappa light-chain enhancer of activated B cells (NF-kappaB) in mice. Hepatol. 2011;54(4):1421-32.

16. Guo C, Xie S, Chi Z, Zhang J, Liu Y, Zhang L, et al. Bile acids control inflammation and metabolic disorder through inhibition of NLRP3 Inflammasome. Immun. 2016:45(4):802-16.

17. Wahlstrom A, Sayin SI, Marschall HU, Backhed F. Intestinal crosstalk between bile acids and microbiota and its impact on host metabolism. Cell Metab. 2016:24(1):41-50.

18. Pineda Torra I, Claudel T, Duval C, Kosykh V, Fruchart JC, Staels B. Bile acids induce the expression of the human peroxisome proliferator-activated receptor alpha gene via activation of the farnesoid $X$ receptor. Mol Endocrinol. 2003;17(2):259-72.

19. Watanabe M, Houten SM, Wang L, Moschetta A, Mangelsdorf DJ, Heyman RA, et al. Bile acids lower triglyceride levels via a pathway involving FXR, SHP, and SREBP-1c. J Clin Invest. 2004;113(10):1408-18.

20. Jiao N, Baker SS, Chapa-Rodriguez A, Liu W, Nugent CA, Tsompana M, et al. Suppressed hepatic bile acid signalling despite elevated production of primary and secondary bile acids in NAFLD. Gut. 2018;67(10):1881-91.

21. Haeusler RA, Astiarraga B, Camastra S, Accili D, Ferrannini E. Human insulin resistance is associated with increased plasma levels of 12alphahydroxylated bile acids. Diabetes. 2013;62(12):4184-91.

22. Dai W, Long J, Cheng Y, Chen Y, Zhao S. Elevated plasma lipoprotein(a) levels were associated with increased risk of cardiovascular events in Chinese patients with stable coronary artery disease. Sci Rep. 2018;8(1):7726.
23. Xiang QY, Tian F, Lin QZ, Du X, Zhang SL, Gui YJ, et al. Comparison of remnant cholesterol levels estimated by calculated and measured LDL-C levels in Chinese patients with coronary heart disease. Clin Chim Acta. 2020;500:75-80.

24. Danese E, Salvagno GL, Negrini D, Brocco G, Montagnana M, Lippi G. Analytical evaluation of three enzymatic assays for measuring total bile acids in plasma using a fully-automated clinical chemistry platform. PLoS One. 2017;12(6):e0179200

25. Chalasani N, Younossi Z. The diagnosis and management of nonalcoholic fatty liver disease: Practice guidance from the American Association for the Study of Liver Diseases. Hepatology. 2018;67(1):328-57.

26. Whelton PK, Carey RM, Aronow WS, Casey DE Jr, Collins KJ, Dennison Himmelfarb C, et al. 2017 ACC/AHA/AAPA/ABC/ACPM/AGS/APhA/ASH/ ASPC/NMA/PCNA guideline for the prevention, detection, evaluation, and Management of High Blood Pressure in adults: executive summary: a report of the American College of Cardiology/American Heart Association Task Force on clinical practice guidelines. Hypertension. 2018;71(6):1269-324.

27. Buse JB, Wexler DJ, Tsapas A, Rossing P, Mingrone G, Mathieu C, et al. 2019 Update to: Management of Hyperglycemia in Type 2 Diabetes, 2018. A Consensus Report by the American Diabetes Association (ADA) and the European Association for the Study of Diabetes (EASD). Diabetes Care. 2020; 43(2):487-93.

28. Task Force M, Montalescot G, Sechtem U, Achenbach S, Andreotti F, Arden C, et al. 2013 ESC guidelines on the management of stable coronary artery disease: the Task Force on the management of stable coronary artery disease of the European Society of Cardiology. Eur Heart J. 2013;34(38): 2949-3003.

29. Qaseem A, Fihn SD, Williams S, Dallas P, Owens DK, Shekelle P. Diagnosis of stable ischemic heart disease: summary of a clinical practice guideline from the American College of Physicians/American College of Cardiology Foundation/American Heart Association/American Association for Thoracic Surgery/preventive cardiovascular nurses association/Society of Thoracic Surgeons. Ann Intern Med. 2012;157(10):729-34.

30. Frommherz L, Bub A, Hummel E, Rist MJ, Roth A, Watzl B, et al. Age-related changes of plasma bile acid concentrations in healthy adults--results from the cross-sectional KarMeN study. PLoS One. 2016;11(4):e0153959.

\section{Publisher's Note}

Springer Nature remains neutral with regard to jurisdictional claims in published maps and institutional affiliations.
Ready to submit your research? Choose BMC and benefit from:
- fast, convenient online submission
- thorough peer review by experienced researchers in your field
- rapid publication on acceptance
- support for research data, including large and complex data types
- gold Open Access which fosters wider collaboration and increased citations
- maximum visibility for your research: over $100 \mathrm{M}$ website views per year
At BMC, research is always in progress.
Learn more biomedcentral.com/submissions 